Revue d'histoire de l'Amérique française

REVUE D. HISTOIRE DE L'AMÉRIQUE FRANÇAISE

\title{
Recherches collectives
}

Chronique documentaire pour une nouvelle histoire coloniale. Les papiers privés et l'Amérique française

\author{
G. A. Chevalier, G. Debien, L. Dermigny, M. Gaucher, C. Hugues, J. Marion, R. \\ Massio et R. Richard
}

Volume 6, numéro 4, mars 1953

URI : https://id.erudit.org/iderudit/301552ar

DOI : https://doi.org/10.7202/301552ar

Aller au sommaire du numéro

Éditeur(s)

Institut d'histoire de l'Amérique française

ISSN

0035-2357 (imprimé)

1492-1383 (numérique)

Découvrir la revue

Citer cet article

Chevalier, G. A., Debien, G., Dermigny, L., Gaucher, M., Hugues, C., Marion, J., Massio, R. \& Richard, R. (1953). Recherches collectives : chronique documentaire pour une nouvelle histoire coloniale. Les papiers privés et

l'Amérique française. Revue d'histoire de l'Amérique française, 6(4), 536-559.

https://doi.org/10.7202/301552ar 


\section{RECHERCHES COLLECTIVES}

\section{CHRONIQUE DOCUMENTAIRE POUR UNE NOUVELLE HISTOIRE COLONIALE}

\section{LES PAPIERS PRIVES ET L'AMERIQUE FRANÇAISE}

Nous publions volontiers cette étude que nous adresse notre actif collaborateur, M. Gabriel Debien. C'est presque un manifeste d'historiens. Les signataires se proposent rien de moins qu'un renouvellement de l'histoire coloniale de la France. Leurs recherches s'orientent surtout vers l'Histoire des Antilles. Histoire qui intéresse notre Institut, dont elle est l'une des provinces, et non dépourvue d'incidences avec celle de la Nouvelle-France en Amérique du Nord. Ce qui nous paraît particulièrement intéressant, c'est la méthode de travail, prônée et déjà mise en auvre par un groupe de chercheurs et d'historiens: travail d'équipe mené vers des sources trop souvent négligées. En lisant ces pages, on pensera aux résultats possibles d'un même système de recherches poursuivi méthodiquement à travers les vieux papiers ou archives - il doit s'en trouver encore en France - des anciennes familles du Canada français si fréquemment divisées entre la colonie et la métropole sous l'ancien régime ou réfugiées dans le royaume après 1760. (NDLR).

Cerner les réalités coloniales d'autrefois, d'hier et d'aujourd'hui, dans l'entrelacs des pistes une à une reconnues, est tâche de longue haleine.' Encore que sujette aux déceptions autant qu'aux coups de chance, elle présente un double intérêt: de marier la connaissance des forces du passé à celle de la société d'aujourd'hui, de faire sentir, de réveiller parfois des continuités familiales, et d'être un service qui se veut commun, pour que lentement s'ébauche à partir d'efforts conjugués une carte humaine, non des densités, mais de l'intensité, de l'aventure et des échanges humains.

Les documents abondent, sans doute; mais, par leur nature ou par suite de mutilations accidentelles ou systématiques, ceux des fonds classés, sinon inventoriés, ne sont pas toujours les plus concrets; souvent même les mailles sont trop grosses et le réel a fui. 
Il en est d'autres qui ne s'offrent point d'eux-mêmes au travailleur: ce sont eux que, sans nulle superstition du rare, nous voudrions mettre au jour. La recherche des papiers de famille nous entraine au contact des milieux et des groupes sociaux, des intérieurs et des styles de vie et, des hôtels urbains aux gentilhommières, des plantations aux entrepôts du négoce, de nos campagnes qui rejettent les "sansterre" ou de nos villes populeuses aux "pays neufs", nous plonge dans des réalités vivifiantes. Ce n'est pas là satisfaction vaine d'explorer et de rassembler de pauvres trésors enfouis au fond des malles ou des armoires, et qui vont s'effritant chaque année, mais plutôt le goût retrouvé de substances nourricières.

La pratique des inventaires n'est pas fuite dans le passé, mais condition du présent. Elle peut en outre, par l'échange entre chercheurs et détenteurs d'archives, par le rapprochement de milieux différents, réussir à rompre le compartimentage faux entre l'érudition et la vie, trop répandu dans l'esprit du "public", et - pourquoi pas à former lentement une opinion éclairée au vrai sens du terme, capable de s'intéresser au recensement et à la conservation de ces éléments du patrimoine national. Dessein ambitieux. Certes. Trop ambitieux? Ce n'est pas sûr, car des expériences répétées montrent de quel intérêt notre entreprise s'est rapidement entourée.

En des fonds de province endormis ou repliés sur eux-mêmes, redécouvrir les grands départs, le labeur et la peine des hommes d'un océan à l'autre, la rencontre et l'affrontement des sociétés, ce n'est pas céder à l'exotisme, mais développer le sens d'une participation au monde, combien plus tonique que l'évocation de souvenirs fanés! Si la malsaine notion d'un passé mort et de "faits historiques", fossilisés dans la mémoire collective, fausse tant d'attitudes contemporaines, suivre au long des vieilles routes maritimes, avec le secret des fortunes et des destinées, les cheminements de la vie et les présences créatrices du temps lointain de l'Empire des Deux Indes, du temps plus proche où l'Europe se mit à la découverte de l'Afrique et de l'Asie, n'est-ce pas retrouver le sens de la continuité humaine? Cette plongée dans le mouvant social révèle, au lieu de temps morts, les séquences de vie.

Point d'illusions cependant! Pour large que soit l'enquête, et si convaincus que nous soyons de son urgence, il n'en faut pas attendre plus qu'elle ne peut donner. Nous savons bien que des documents 
de ce genre, correspondances, papiers d'affaires, comptabilités, sans lesquels une histoire "totale" resterait un rêve, ne sauraient, en revanche, suffire à l'élaboration des synthèses nécessaires. Dans l'immédiat, l'objectif est plus limité; il s'agit de présenter les grands problèmes sociaux liés à la colonisation - esclavage, condition des gens de couleur, types d'exploitation, aspirations à l'autonomie, etc., - de faire surgir tel réseau d'intérêts encore peu connu, et de préciser le financement des entreprises coloniales par des monographies de quartiers de familles, de plantations, ou simplement d'individus. Par la suite, tout ce travail d'approche pourra être utilisé, ces documents précis plus minutieusement et attentivement interrogés pour aborder l'étude des aspects et des conséquences des contacts de civilisations.

Ce ne sont que les premiers et modestes résultats que nous rassemblons ici, en souhaitant qu'ils suscitent échanges, critiques et apports. C'est le vœu de ceux-là mêmes qui, nombreux déjà, nous ont aidés, qu'en toute région se multiplient les correspondants et qu'à la dispersion des sources réponde la liaison des efforts, afin que nous progressions ensemble. Dans nos recherches tout a été et reste fondé sur l'entr'aide. Nos dettes sont immenses: nos remerciements ne sauraient tout acquitter. Saurons-nous même citer ici toús ceux qui nous ont conseillés, qui ont répondu à nos questions et qui nous ont largement ouvert leurs papiers, leur érudition et leurs amitiés? C'est un plaisir pour nous d'assurer de notre gratitude Madame Balguerie, Madame Pierre Brunel, Madame la vicomtesse Guy de Vanssay, Madame du Vigneau, Madame la marquise de La Prunarède, $M$. le baron Anduze de Saint-Paul, M. Raymond Durand, le docteur Sabatier, M. Jean Giry, M. le comte de Mondion, M. Henry de Marcillac, M. Bordères, M. de Quincerot, M. de la Merveillère, $M$. le $C^{\text {el }}$ de Brach, M. de Laulanié, M. le vicomte de Maupeou, M. Gayon, M. le $C^{d t}$ de la Rochefordière, M. Ch. Blanc, M. l'abbé Gardey, M. le comte de Gastines, $M$. le chanoine de Laugardière, M. Marchegay, M. Pilastre, M. le $C^{d t}$ David, le docteur Picheire, M. Jean Fouchard, M. Denys de Bonnaventure. Il sera facile de voir, chemin faisant, leur part dans nos recherches communes.

MM. les archivistes aussi ont dirigé ou allégé notre tâche. Ils nous ont fait connaître des pistes, ouvert accès à des documents ignorés, rendu facile la communication de gros dossiers. MM. Delafosse, 
en Charente-Maritime, Salvini, dans la Vienne, MM. Cravayat, dans le Cher, Avezou, dans l'Isère, M. Gouron, dans l'Hérault, MM. les archivistes de la Sarthe, de l'Indre-et-Loire, de la Loire et de la Haute-Loire nous ont montré par mille faits qu'obliger vite c'est obliger deux fois.

Afin que nous progressions ensemble... "Nous" doit s'entendre au sens plein, d'équipe tâtonnante peut-être, mais de bonne volonté, d'équipe ouverte qui n'a d'autre ambition que de "communiquer" avec les hommes et avec les choses. La mise en commun d'indications, de trouvailles et de problèmes procède à la fois d'un souci d'efficacité et du besoin d'un climat; plutôt que collaborateurs, expression affadie, participants d'une même œuvre, ...tels qu'en eux-mêmes enfin le service de l'Histoire changerait des historiens.

\section{$*^{*} *$ \\ I. - Vers l'Amérique}

Bien des facteurs se conjuguaient aux XVIIe et XVIIIe siècles pour inciter au départ jeunes gens et hommes faits: un élément intellectuel, l'impression d'une lecture sur un adolescent parfois, mais essentiellement le besoin de refuge ou d'évasion, le fait de religion, les revers de fortune ou l'espoir de montée sociale, et tout le "surplus" exportable de familles prolifiques. Plutôt que d'en dresser une liste, qui serait interminable, contentons-nous d'analyser un exemple régional, celui du Languedoc.

Bien qu'apparemment en retrait des grandes routes océaniques, cette province a entretenu des relations assez suivies avec les Amériques, soit par Sète qui armait de temps en temps pour les îles, entre 1717 et 1735 notamment, soit par Marseille ou Bordeaux. Ces liaisons, sans atteindre à la densité de celles que nouaient les pays de l'Ouest, montrent néanmoins que l'aventure et le commerce aux Antilles surtout, mais au Canada aussi, intéressaient vraiment toutes les parties du royaume - même les plus reçulées, comme la montagne lozérienne. Telle est l'impression qui se dégage d'une enquête seulement ébauchée.

Ces relations sont de trois sortes. 


\section{I. - Le Refuge}

D'abord, l'émigration, en partie temporaire, de gens qui, en difficulté en France, lèvent le pied et vont se refaire une virginité sous le tropique. Dans les bilans ${ }^{1}$ ou les minutes notariales revient fréquemment la mention "un tel failli, disparu, ou parti à l'Amérique" qui indique que la pratique de "passer aux isles" pour se faire oublier est réellement entrée dans les mœurs.

Parfois cela se rehausse d'escroquerie pure et simple, comme dans le cas de Charles Deidier, commis de Coulomb, négociant à Montpellier, qui, envoyé en 1777 par son patron en tournée sur la frontière de Catalogne, passe à la Martinique avec l'argent de sa recette $^{2}$. Ou dans celui de ce Dessombs, marchand de Sauve (Gard), qui achète dans son pays des bas et étoffes de soie, et à un négociant de Montpellier pour 1000 écus de liqueurs et de parfumerie, le tout payable en 1778 au retour de la foire de Bordeaux, et qui s'embarque froidement pour le Cap-Français, afin d'y débiter sa pacotille et de ne pas payer ses dettes. Pour brouiller la piste, car naturellement ses creanciers portent plainte auprès des magistrats de Saint-Domingue, il change plusieurs fois d'identité, si bien qu'on en est réduit à chercher sa trace, du Cap aux Cayes, d'après les marques et étiquettes des caisses de marchandises qu'il a emportées ${ }^{3}$. Le ton des requêtes montre qu'il ne s'agit pas de cas isolés.

La prévarication peut d'ailleurs s'y associer: Garraud, originaire de Foix, est garde-magasin au Canada pendant la Guerre de Sept Ans; à l'instar de l'Intendant Bigot ${ }^{4}$ il y fait sa pelote, consistant en quatorze lettres de change tirées par le trésorier de Québec sur les trésoriers de la Marine à Paris, et toutes passées sous des noms d'emprunt. Il apprend à son arrivée à Bordeaux que le paiement du papier du Canada est suspendu et qu'on instruit le procès des tripoteurs; craignant d'être arrêté, il s'efforce en hâte de négocier ses lettres, qu'un négociant toulousain finit par lui prendre à 50 ou

1. A.D. Hérault, Série B, Juridiction consulaire.

2. No 225 de cette série.

3. Papiers de M. Raymond Durand, 8 , rue de la République à Montpellier.

4. Voir plus loin, p. $\mathbf{5 4 5}$. 
$60 \%$ de perte, achète quelques marchandises avec cet argent et file à Saint-Domingue, où il mourra bientôt ${ }^{5}$.

Cette "habitude" sera encore illustrée après la Révolution, par Victor Embry par exemple. Célui-ci, fils d'un des principaux négociants d'Agde, gendre de Balguerie, autre négociant, est un fainéant prétentieux qui se fait appeler d'Embry; fonde à Paris sous l'Empire des maisons de commerce fictives en abusant du nom de son père, et pour finir dilapide son héritage. En 1814, muni d'une pacotille de cent napoléons extorqués à son beau-père, il s'embarque à Bordeaux pour la Martinique, en compagnie "d'une femme perdue" qui s'appelle tantôt comtesse de B..., tantôt Mrs. Rodgers. Nommé encanteur d'office au Fort-Royal en février 1819, il est obligế de déguerpir dès le 31 mars, laissant une dette de 3.775 francs envers le Trésor; il se rend aux Etats-Unis, puis à la Guadeloupe, d'où il rentrera en France en 1821. En 1824 il sera commis aux écritures à $1000 \mathrm{fr}$. par an chez un négociant de Montpellier ${ }^{6}$.

\section{2. - L'ÉtA BLISSEMENT}

Mais l'Amérique n'est pas que le refuge des mauvais garçons. Second type d'émigration, honnête celle-là, c'est le fait de gentilshommes, de bourgeois ou de petites gens, des ports, des villes marchandes ou du haut pays, qui vont tenter fortune: Meynadier, négociant et planteur caféier à Jérémie (plantation de Mont-Soucy sur les hauteurs des Irois ${ }^{7}$; Antoine-Guillaume Lemire, de Sète, établi à la Grande Anse; Pierre Sérane, Sétois lui aussi, négociant à Jérémie où il épouse Rosalie Piron "originaire du canton des Abricots", quartier caféier; il rentrera mourir a Sète, et sa veuve se remariera avec Armand Rousson qui armera pour Saint-Domingue ${ }^{8}$; André Bérard, d'Alès, négociant au Cap et propriétaire, avec sa femme, Marguerite Riboutet, d'une petite caféière de 140.000 livres à la Marre-à-la-Roche ${ }^{9}$; le baron de Sainte-Croix, originaire de SaintJean de Gardonnenque, lieutenant de roi à Léogane, marié à Mlle

5. Papiers Anduze de Saint-Paul, chez M. le baron Anduze de Saint Paul, a Montpellier, 11, rue Rondelet.

6. Papiers Balguerie, chez Mme Balguerie, place de la Marine, à Agde.

7. et 8. Etude Blanc à Sète: minutes Taissié (reg. 6) et Lardat (reg. 3 et 4)

9. Papiers Anduze de Saint-Paul. 
Cottin, riche créole du quartier, qui commande au Môle en 1789, puis le cordon de l'Ouest au début de l'insurrection des esclaves $(1791)^{10}$; Henri de Lavit, de Clairac près de Bédarieux, ancien officier de cavalerie devenu planteur au Petit-Goave et commandant des quartiers du Dondon et de la Grande-Rivière dans le Nord, dont une fille épouse Lory de la Bernardière, originaire de Nantes, greffier de la sénéchaussée du Petit-Goave, puis négocian ${ }^{11}$; Jean Duquesnot, de Nîmes, conseiller au Conseil Supérieur de Léogane (1686-1711) Jean-Jacques Carles, de Montpellier, avocat au Cap en 1785'3; Vallat de Saint-Charles, colon caféier à Plaisance et Pilate, qui se retire à Lodève ${ }^{14}$; Grandier, de Carcassonne; Barthélemy Salager, négociant à la Guadeloupe ${ }^{15}$; Lalibert, d'Esperaza, chirurgien à Ouanaminthe (province du Nord) ${ }^{16}$; Dorville, Fourniol et Lasserre, de Limoux, fixés à la Martinique ${ }^{17}$, etc.

Du côté lànguedocien, la région de Limoux-Espéraza, en particulier, semble avoir été un foyer d'émigration vivace, dont la présence comme fonctionnaire à la Martinique en 1826 du fils de Jean Isard, cultivateur à Espéraza, souligne la tradition ${ }^{18}$. Du côté américain, un double trait marque ce mouvement. D'une part, une prédilection pour Saint-Domingue et, à Saint-Domingue, pour ces quartiers encore de l'Ouest, mais si proches du Sud: Léogane et le Petit-Goave - bien que l'on trouve aussi des Languedociens établis dans la région du Cap, une des plus anciennement colonisées et des plus évoluées. D'autre part, la tendance des fils à dévorer en France la fortune amassée par les pères. Ce que fait Omer Martin, "Ameriquain d'Adge", qui tombe dans la main des usuriers et accumule les dettes: 90.000 livres en quelques années, alors que son bien à SaintDomingue est évalué à 200.000; ou le fils de Lavit, qui, rentré à Béziers, a tôt fait de manger le produit de la vente de l'habitation

10 A.D. Hérault, C 155.

11. Papiers du Dr Sabatier, a Montpellier, 31, rue St-Guilhem.

12. Papiers Galbaud du Fort, chez Mme la comtesse du Fort, à Angers, 59, rue de Brissac.

13. Papiers Carles, Bibliothèque de Saint-Lo, Ms. 154.

14. Journal des Colonies, XIV: 46-47.

15. Papiers Bérindoague, chez Mme la comtesse du Fort.

16. Papiers Anduze de Saint-Paul.

17. Papiers de M. Jean Giry à Béziers, 26, rue Française.

18. Papiers Anduze de Saint-Paul. 
laissée par son père, et, acculé à la ruine, vit avec sa femme et ses cinq ou six enfants aux crochets de sa belle-mère, Mme de Solignac, sur les revenus de la terre qu'elle possède à Magalas ${ }^{19}$.

\section{3. - Les MARIAges CRÉOles}

Troisième type de lien, enfin, c'est le cas des gentilshommes qui deviennent propriétaires à Saint-Domingue en épousant des héritières créoles: mariage à Tours en 1772 du comte de La Prunarède, lieutenant-colonel de cavalerie, âgé de 42 ans, avec Marie-Gabrielle de Maupoint, orpheline de 14 ans, à peine sortie du couvent de Panthemont, propriétaire de deux caféières à la Grande-Rivière, léguées par son père qui était receveur des octrois au Cap ${ }^{20}$; mariages, en 1791, du marquis de Grave, de Pézenas, avec Mlle de la Toison de Rocheblanche, née à la Grande Raque (Saint-Domingue) et dotée, paraît-il, de 400.000 livres de revenu; en 1790, à Paris, du marquis de Barbeyrac-Saint-Maurice, député à la Constituante, avec "Bonite" Colheux de Longpré, fille d'un conseiller au Conseil du Portau-Prince, colon à Léogane, qui lui apporte en capital environ 100.000 livres argent des colonies, et en revenu, quelque 22.000 livres provenant des sucreries Longpré à Léogane et La Rochejacquelein au Baconnais (Nippes), de l'habitation Bernardon au Cul-de-Sac, et de deux caféières au Grand-Coave. Propriétaires non-résidents, cela va sans dire, même dans le cas de La Prunarède, qui ne fait à SaintDomingue qu'un voyage de prise de possession et s'en remet ensuite à des gérants.

\section{II. - A l'Amérique: Les COLONIES SECONDAIRES}

Bien qu'elles aient été orientées avec le même zèle et la même méthode vers toutes nos colonies d'Amérique, nos recherches n'ont pas donné des résultats uniformément encourageants. Si pour SaintDomingue, l'île reine, et à notre surprise, pour la Guyane, notre récolte est abondante, pour le Canada, la Louisiane et les petites

19. A.-D. Hérault, C 155.

20. Arch. Nat., Colonies, E 307. 
Antilles la pauvreté de notre cueillette est extrême. Il est à ces différences des explications très simples: le Canada a été perdu avant les années des grandes sorties de capitaux vers les colonies, et l'incendie de Saint-Pierre a détruit à la Martinique en 1902 bien des papiers. Mais on comprend mal pourquoi nous avons seulement quelques dossiers pour la Louisiane et pour la Guadeloupe. Sans doute que la prospection des sources de l'histoire des Antilles qui sont demeurées françaises demande une méthode un peu différente de celle que nous avons suivie. Il faut partir plus systematiquement de la situation présente, de l'histoire des affaires, de celle des sociétés agricoles, des raffineries, des maisons de commerce et des banques, et établir d'abord notre réseau de recherches dans les îles mêmes.

Dans les familles on trouve assez peu de documents sur le Canada, et beaucoup de ceux que l'on conserve ont été publiés ou ont servi à des études historiques. Les souvenirs se concentrent plutôt autour des portraits.

Il en est au moins trois à Artigny, près Loudun (Vienne) chez M. le comte de Mondion qui illustrent l'histoire d'une famille du Canada, la famille de Ganne: le portrait d'un archiprêtre de Québec, celui d'un chevalier de Saint-Louis, enfin celui d'une demoiselle de Ganne qui épousa Charles-César de Mondion"2.

Dans les papiers A. Richard, légués aux Archives de la Vienne, deux documents isolés intéressent l'histoire des guerres au Canada au milieu du XVIIIe siècle ${ }^{22}$ :

10) le "Récit du combat naval du 19 et 20 janvier 1741 en Amérique entre Français et Anglais, tiré de la relation écrite par un officier pour M. Hardy", 4 p.

2o) "Événements de la guerre au Canada depuis le 19 septembre 1759 jusqu'au 14 juillet 1760", $14 \mathrm{p}$.

Nous n'avons pu nous assurer si ces narrations étaient inédites.

21. Un Georges de Ganne fut capitaine au gouvernement des Troig-Rivières au XVIIe siècle.

22. Papiers A. Richard, 61 bis. 
Alfred Barbier avait en 1900 écrit l'histoire d'Un munitionnaire du roy à la Nouvelle France ${ }^{23}$, grâce aux papiers de Joseph Cadet. C'était surtout l'histoire des démêlés de ses héritiers avec les bureaux de la Marine qui entendait faire main basse sur la succession. Ces papiers sont curieux pour suivre en France la mise en place de la fortune que Cadet avait, disons, acquise, au Canada (1752-1788). Ils sont maintenant aux Archives de la Vienne ${ }^{24}$ et seraient à revoir de près depuis que $M$. G. Frégault $a$, par ses travaux sur l'intendant Bigot, refait l'histoire de ces campagnes de spéculations que furent aussi les années de la guerre de Sept Ans au Canada.

Par un autre don récent les papiers de la famille Letard de la Bouralière sont entrés aux Archives de la Vienne. Ils viennent d'être classés. Leur intérêt n'est pas seulement poitevin. Par son remariage avec un Letard de la Bouralière, Marie-Anne Saugy, veuve de Vincent Condonneau du Jacquelin, ancien officier d'infanterie à la Louisiane, fit entrer dans sa nouvelle famille une succession coloniale, c'est-à-dire quelques biens et beaucoup de soucis. Une correspondance du temps de l'Empire, venue de la Nouvelle-Orléans, exprime les inquiétudes et les espérances d'Amérique ${ }^{25}$.

Aux mêmes archives, encore, entrés aussi depuis peu, d'autres papiers relatifs à des affaires de Louisiane $(1802-1813)^{26}$.

Enfin il faut faire ici un écho aux papiers Collette, qui seront examinés plus loin. Plusieurs lettres datées de la Nouvelle-Orléans décrivent les embarras, la vie nouvelle d'un grand blanc créole de Saint-Domingue qui a préféré chercher un refuge en Louisiane avec quelques-uns de ses esclaves plutôt que de venir en France qu'il connaît à peine ${ }^{27}$.

Certaines parmi les petites Antilles qui furent colonies françaises sont des domaines négligés de l'Histoire. C'est le cas de Sainte-Lucie:

23. Mémoires de la Société des Antiquaires de l'Ouest, 1900.

24. J. 158.

25. J. Papiers Letard de la Bouralière.

26. J. 157.

27. Chez M.G.A. Chevalier, à Alger, 2, rue Colbert. 
les colons y étaient peu puissants et les négociants y servaient, pour les îles plus importantes, la Guadeloupe, la Martinique, Saint-Domingue, d'intermédiaires à l'interlope étranger, commerce qui reçut droit de cité et fut réglementé à partir de 1771. Tout ce qui pourrait être recueilli touchant Sainte-Lucie serait du plus grand intérêt; nos recherches ne nous ont mis en présence que d'un très mince dossier, sans relief, presque sans couleur, et son analyse fera sentir le besoin que nous aurions d'une documentation plus riche, plus étendue.

La très courte aventure d'Antoine, François de la Buxière à Sainte-Lucie, - il n'y séjourna que de janvier au 3 mars 1764, date de sa mort, en qualité d'Inspecteur des Magasins royaux de la colonie - n'est intéressante que par son encadrement. Fils d'un avocat fameux de Bourges qui s'était patiemment, au cours de 35 ans, constitué une solide fortune foncière, La Buxière n'avait mené, jusqu'à la mort de ses parents, qu'une existence médiocre; en 1744, âgé de 28 ans, il n'était que soldat au régiment de Monaco-Infanterie. En 1747, moins d'un an après le partage de la succession de ses père et mère, dont il recueillait le quart, La Buxière épouse Charlotte Perrichon, fille d'un paysan cossu. Rapidement trois enfants sont nés. Mais La Buxière reste un homme peu enclin au travail et à l'économie: en 1758, il a perdu l'emploi un moment tenu dans les gabelles, son avoir est dissipé et passé partie aux mains de ses créanciers, partie dans celles de sa femme, de lui séparée de biens par sentence rendue au bailliage de Bourges. C'est probablement grâce à l'appui de son frère aîné, Thomas, avocat à Paris, que La Buxière peut subsister jusqu'en 1763 et qu'il peut obtenir alors commission d'Inspecteur des Magasins royaux à Sainte-Lucie.

Quels espoirs le partant fondait-il sur ce poste colonial? Sans doute y voyait-il le dernier moyen de reconquérir quelques biens, le dernier essai possible à un homme de 48 ans. Il emmenait ses deux fils Joseph et Robert, autre créance sur l'avenir. Mais bientôt, l'Intendant de Sainte-Lucie, Marc-Antoine Chardon, envoyait la nouvelle du décès de La Buxière, survenu au Carénage le 3 mars 1764; le certificat délivré attestait, qu'inventaire des meubles et objets du défunt avait été dressé "par le sieur Guéroard, écrivain de la Marine, seul officier de plume breveté de Sa Majesté, qui se trouvait pour lors dans la colonie, assisté du sieur Fertard commis aux écritures, 
faisant les fonctions de contrôleur de la Marine, à cause du décès du sieur Martin de Saint-Philippe nommé par le Roy à ladite place". Il semble que le climat de Sainte-Lucie ait été particulièrement meurtrier en ce début de 1766 pour les fonctionnaires de Sa Majesté.

Qu'advint-il de Joseph et Robert de La Buxière? On le discerne mal. Ils n'assistèrent point leur père dans ses derniers moments. Déjà ils l'avaient quitté. La discorde régnait-elle entre eux? On retrouve plus tard Joseph soldat au régime de Chartres, auquel il appartenait lors de sa mort à l'hôpital militaire de Sedan en 1774. Quant à Robert, toutes les recherches poursuivies à son sujet pendant quinze ans par sa famille furent infructueuses. Le 20 juin 1777 , le frère Lacombe, religieux Augustin, curé du Carénage, écrivait à son couvent de La Rochelle: "On m'a assuré qu'il était mort [ dans les îles anglaises ], mais il ne m'a pas été possible d'en tirer un acte mortuaire, vu qu'il n'y a pas de mission ni de curés dans ces pays-là et que les Français pauvres et isolés qui ont le malheur de tomber malades et de mourir, ne sont pas plus regardés que des chiens. Si on les enterre, ce n'est que parce que l'odeur cadavreuse (sic) les infecte. Jamais acte mortuaire n'en paraît à moins qu'ils ne laissent de quoi payer leur enterrement au ministre anglais ${ }^{28}$."

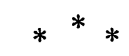

Pour la Guadeloupe on a grand'peine à trouver des correspondances privées, des dossiers de plantation, des papiers de commerce et d'affaires. Ils sont rares et fragmentaires. Et ce qui est vrai des XVIIe et XVIIIe siècles l'est davantage encore du XIXe siècle, qui est aux Antilles, et souvent dans la métropole, le siècle de la documentation difficile. Nous sommes donc heureux de dire quelques mots des papiers d'affaires de la Guadeloupe, abordés cet hiver. Ils intéressent à la fois l'histoire du commerce bordelais et celle dı commerce à la Pointe-à-Pitre.

Près de Vic-en-Bigorre, sur la route de Tarbes est le domaine de Saint-Onis. Y vivait au XVIIIe siècle noble Eugène de Lataste, seigneur de Pouey. Le domaine fut vendu en 1823 par la dernière

28. Papiers La Buxière, chez M. Henry de Marcillac, à Valencins, par SaintFlorent sur Cher (Cher). 
Lataste à Jean Brescous, planteur à la Lézarde, quartier du PetitBourg, à la Guadeloupe, qui décède en 1826 à la Pointe-à-Pitre. Il était en même temps négociant. Il dut laisser une situation embarrassée, car sa plantation fut vendue par licitation au profit de René Claudin, et Saint-Onis passa au même moment, vers 1830, à Bertrand Cestia, négociant à la Pointe-à-Pitre. Il est probable que Brescous et Cestia étaient bigourdans. Par héritage Saint-Onis est venu des Cestia aux Bodères, de Vic-en-Bigorre, qui l'ont toujours.

Dans le grenier de Saint-Onis, une courte après-midi d'hiver a permis de reconnaitre deux malles pleines à craquer de vieux papiers. Une seule a été vue. On ne peut même pas parler d'exploration. Tout est à classer, mais d'abord à vider. Ce premier coup d'œil a déjà distingué:

10) un grand cahier de copies de lettres datées de La Pointe-àPitre, du 28 mars 1827 au 14 aout 1828. Il a 101 pages et compte environ 150 lettres adressées par la maison Cestia de La Pointe-àPitre à des colons de la Guadeloupe, à des commerçants des îles voisines et à des maisons de commerce de Bordeaux. Cette maison qui prit la suite de la maison Despalangues, faisait des affaires surtout avec Bordeaux, achetant des vins, cognac, vinaigre, du fromage, du mals, des farines, des vêtements de confection pour les nègres et surtout de la morue. Les achats se font aux Laville, Despalangues, Martin, Estiboy, Turettes et Bordères; au Havre à Bauche. C'est avec Porto-Rico et Saint-Thomas que sont entretenues aux Antilles les correspondances les plus actives.

On retiendra sans doute d'abord les lettres adressées a la maison Despalangues au sujet d'une "exploration de la côte d'Afrique", par le brick l'Éclair, armé a Saint-Thomas en octobre 1827. Entendez une expédition négrière. Le bâtiment muni de 8 canons et de 25 hommes d'équipage doit ramener 350 têtes. Jamais le mot esclaves, non pour être "à l'abri de toute recherche en cas d'événement", mais par une sorte de pudeur de style, car tout le reste est en clair dans ces lettres. Le retour est attendu pour janvier ou février 1828. La mise dehors monte à 130.000 francs, 60.000 pour le corps du navire, 70,000 pour la cargaison. L'assurance sera à $30 \%$. On ne dit point le tonnage.

Précédemment 312 esclaves avaient été traités en quinze jours, et 110 autres même en cinq jours. Cela nous donne une idée de la pré- 
paration et du choix méthodique des points d'embarquement. Au XVIIIe siècle, la traite libre était souvent un lent ramassage des captifs d'un poste à l'autre. Désormais surveillée d'assez près la traite est organisée comme un enlèvement rapide.

L'Eclair est capturé le 2 février 1828 par une croisière française; "la cargaison" était à terre. Il fallut envoyer des îles un autre navire, vide, qui ramena, non 350 esclaves, mais 300 .

2o) Un gros dossier de 4 à 500 lettres, reçues à la Pointe-à-Pitre par la maison Cestia entre 1823 et 1830, toutes d'intérêt commercial.

Ce sondage propose l'entreprise d'une étude de longue haleine, celle d'une maison de commerce à la Guadeloupe sous la Restauration.

Se rapportant à la Martinique, signalons d'abord en annonçant sa prochaine exploration méthodique, un très riche dossier de plantation, au milieu des papiers de la très vieille maison de commerce bordelaise des Gradis ${ }^{29}$. Ce qui fait la rareté des documents de ce dossier c'est qu'ils dépassent les années de la Révolution et conduisent l'histoire des cultures et des esclaves jusqu'au moment de l'émancipation.

Les autres documents martiniquais qu'on nous indique, sont surtout d'interêt militaire ou politique:

10) Une lettre écrite de la Martinique en 1762, après la conquête de cette île par les Anglais, 13 p. ${ }^{20}$

20) Un lot de 25 documents émanant du général sir Ralph Abercromby, tous datés de la Martinique (1796) après l'occupation de l'île par les forces Anglaises. Ce sont surtout des comptes et des ordres ${ }^{31}$.

3o) Une "statistique militaire pour 1880. Mémoire sur l'état des routes militaires, des armes et munitions sur l'emplacement des batteries, les mesures à prendre pour la défense générale. ${ }^{32}$ "

29. 1, rue Condé, Bordeaux.

30. Archives de la Vienne, Papiers A. Richard, 61 bis.

31. Catalogue no 356 de la librairie Myers, de Londrea, 1949, No 4.

32. Archives de la Vienne, F. 3. 
4o) Un mémoire judiciaire imprimé en 1816 sur les affaires d'Eugène J. Stanislas Foulon d'Ecotier, ancien Intendant à la Martinique ${ }^{33}$.

La Guyane, bien qu'elle fût englobée dans les "Isles", était en marge du monde caraïbe. Elle resta une zone très particulière, économiquement faible, pour des raisons multiples, mais surtout par le manque de capitaux, constamment ressenti ${ }^{34}$. Cependant la méfiance extrême des bailleurs de fonds pouvait tomber devant la perspective d'une affaire vraiment saine, capable de donner d'extraordinaires bénéfices: bien renseignés, il semble que ce soit sur une telle certitude que se fondèrent, en 1787, les acquéreurs de l'habitation Beauregard à Cayenne, provenant de la liquidation, entamée en 1763, des biens des Jésuites à la Guyane ${ }^{35}$. Au départ d'un dossier de 403 pièces concernant cette habitation $(1787-1876)^{36}$, se trouve le versement de 16,327 livres 10 sols effectué par l'un des participants, Jean, François, Marie Rouhette de Villeclos. Ainsi est établi, année par année, le compte des profits de ce capital; pourtant ce dossier de comptabilité fait aussi plonger dans la vie de la plantation, image réduite mais fidèle de la Guyane; enfin il offre, de 1817 à 1829, des précisions, rarement rencontrées ailleurs, sur la part du revenu colonial prélevée par l'État et par le commerce.

En 1787, pour la somme totale de 280.000 livres, étaient cédés à Bertrand Bajon, seul en nom à l'acte de vente, les biens suivants: un ensemble couvrant la superficie de 990 carrés (environ un millier d'hectares), situé au quartier de Remire, et représentant, moins la

33. Id. J. 160.

34. Voir l'essai à paraître: R. Richard, Une énigme d'histoire coloniale: la Guyane française.

35. Le 20-IX-1787, à Paris, devant Me Doillot, notaire séquestre de l'Union des créanciers des Jésuites (dont les syndics généraux étaient Jacques, François Limay, intéressé dans les affaires du Roi, demeurant rue Montmartre à Paris, J.B. Vandenyvert, banquier rue Vivienne, et Jean, François Matbedat, directeur de la Caisse d'Escompte). Le dépouillement des minutes de l'étude Doillot permettrait de retracer l'histoire de la liquidation des biens des Jésuites.

36. Papiers Dumont-Quincerot, chez M. de Quincerot, 4, Place George Sand à Bourges (Cher). 
partie vendue le 2 mai 1781 à MM. de Chambly, les trois anciennes habitations mitoyennes appelees Loyola, Mont-Louis et Beauregard, que possédaient les Jésuites en ce lieu; ces terrains portaient des plantations de cannes, de coton, de cacao et de manioc, toutes les installations nécessaires à une grande sucrerie, un nombreux cheptel et 372 esclaves; à 12 lieues de là, au quartier de Roura, le long de la rivière Oyack dite aussi de la Comté, et près de son embouchure, s'ajoutait une étendue de "bois debout", évaluée fictivement à 600 carrés, contenue avant l'expulsion des Jésuites dans leur habitation Saint-Régis, dont les terres défrichées avaient été vendues le 29 décembre 1785 à La Fayette ${ }^{37}$.

Que le prix de vente fût fixé à 280.000 livres peut étonner. Moins, si l'on songe qu'il s'agit d'une habitation guyanaise; moins encore si l'on connaît les difficultés éprouvées par les créanciers des Jésuites pour tirer quelque argent de ces biens depuis 1763. Le fils de l'un des syndics de ces créanciers, Gaétan Prépaud, avait été envoyé en 1764 avec mission d'établir un inventaire général et de procéder à la mise en vente. De l'ensemble des biens estimés par lui 1.500.000 livres, rien n'avait pu être vendu en un $a^{33}$. Il fallait, pour mettre fin à l'agitation née à Cayenne des rivalités et des convoitises, que l'acquéreur de la presque totalité fût, en février 1766, Prépaud lui-même, associé à l'un de ses frères. Mais les conditions à eux consenties étaient

37. Avec cette ancienne sucrerie, La Fayette acquérait la "ménagerie" de Maripa, autrefois domaine d'élevage des Jésuites, en amont sur l'Oyack. L'envoi de La Fayette en possession de ces biens et de 48 esclaves en dépendant n'avait pas encore eu lieu en septembre 1787, malgré la présence à Cayenne, depuis un an, de plusieurs fondés de pouvoirs successifs; le premier d'entre eux, Henry de Richeprey, avait pour mission de réaliser l'émancipation progressive des esclaves achetés par La Fayette: devant les résistances locales, cette expérience dut être poursuivie, et seulement grâce à l'appui de l'Ordonnateur Lescallier, sur une autre acquisition faite en 1786, l'habitation La Gabrielle au quartier de Remire. Richeprey ayant été emporté par les fièvres en février 1787, on ne sait quelle suite fut donnée à cette tentative. Les discussions qui s'engagèrent en France dès le début de la Révolution autour de La Fayette, ne permettent pas de savoir si, comme le prétendent ses détracteurs (cf. Arch. Nat., Dxxv 86, pièce datée du 28-XII-1789), il décida lui-même d'y mettre fin en proposant en juin 1789 la vente de ses esclaves, ou si, comme l'assurent un frère de Richeprey en 1791 (cf. Mercure Universel, 14-V-1791, p. 213) et La Fayette dans ses Mémoires (t. V, p. 179, de l'édit. Tournon aîné, 6 vol., Paris, 1836-1838), l'essai continué ne fut brisé que par le séquestre révolutionnaire, après le 13 août 1792. Le mérite d'avoir attiré l'attention sur ces questions revient à $M$. H. Guilhamon, dans son introduction à l'édition des Voyages en Haute-Guienne de Richeprey.

38. Cf. Ch. Larère, S.J., La suppression de la mission de la Guyane française (1763-1766), Archivum Historicum Societatis Iesu, vol. IX, 1940: 208-226. 
extraordinairement favorables: probablement pour moins de 400.000 livres leur étaient abandonnées les diverses plantations, réserves de bois, ménagerie déjà mentionnées, chargées alors de 799 esclaves. Sûrs de pouvoir s'acquitter, les frères Prépaud avaient décidé d'édifier une gigantesque entreprise, portant l'atelier dès avril 1766 à 1022 têtes, construisant de vastes bâtiments, multipliant les pièces de cannes. Mais l'effort exigé de tous, les dépenses inconsidérées, les contretemps et la maladie avaient ruiné cet immense domaine. En 1779, les frères Prépaud étaient tous deux décédés, laissant leur dette impayée et seulement quelque 500 nègres. Tout avait été remis aux mains de l'union des créanciers des Jésuites, qui avait dû faire administrer par un gérant.

En 1787, celui qui était en place, Bertrand Bajon, originaire des environs de Toulouse, ancien chirurgien-major de l'Ile de Cayenne, pensionnaire du Roi, vint à Paris et acheta ces biens coloniaux dont il avait parfaite connaissance. En 5 à 6 mois de séjour parisien Bajon avait pu nouer les relations nécessaires à la réunion des capitaux exigés: l'acte de vente est précédé de diverses conventions aux termes desquelles est constituée une société où Bajon, qui continuera la gestion à Cayenne, figure pour 1/2, Georges Leroy de la Brière, demeurant à Paris, pour $1 / 4$, et Rouhette de Villeclos, fils de celui qui a dû faciliter les négociations, Me Théodore Rouhette, écuyer, ancien avocat au Parlement de Paris, pour 1/4. A Beauregard et ses dépendances s'ajoutait une maison, rue de Choiseul à Cayenne, acquise de la succession Prépaud 5.310 livres, pour servir de comptoir et d'entrepôt, et dont chacun régla aussitôt sa part.

Pour ceux qui sont en France, l'histoire du revenu jusqu'en 1805 se confond à peu près avec celle du paiement de Beauregard. 60.000 livres avaient été versées au contrat. Les 220.000 livres restant devaient se solder en 4 échéances avec intérêts a $4 \%$, la dernière au 1er janvier 1791. Malgré la vente en 1789 de l'ancienne plantation Loyola (151 carrés) pour 28.905 livres au négociant de Cayenne Etienne Franconie ${ }^{30}$, il restait dû, capital et intérêts, 52.888 livres

39. On remarquera a cette occasion que les terraing de pentes et de hauteurs,sur la montagne de Mahury, étaient prisés 3 fois la valeur des terrains plats, mal drainés sans doute ( 300 livres contre 100, le carré. Minutes Paguenaut, notaire a Cayenne, 9-V-1789). 
au ler pluviose An II. Quittance finale dut être obtenue avant 1796 année où l'on voit paraître les premiers revenus. Mais à cette date, Bajon ne peut empêcher le séquestre de la moitié revenant à des propriétaires absents; jusqu'à la fin de 1804, 60.831, Fr. 46(monnaie coloniale) seront ainsi retenus par la Caisse de la Marine, dont la liquidation ne donnera en France que deux inscriptions de rente $5 \%$, l'une de $355 \mathrm{Fr}$. en 1805 , l'autre de $774 \mathrm{Fr}$. en $1820^{40}$. Cette période 1787-1804, où tant de propriétaires coloniaux avaient perdu leur bien, se solde ici par un bénéfice délicat à évaluer, à cause des délais de recouvrement, mais considérable, puisqu'il y entre en compte l'amortissement $\mathrm{du}$ fonds.

La phase suivante de l'exploitation, de 1805 a la fin de 1816, est encore marquée de graves perturbations. En 1805, aucun des associés n'était plus à Cayenne: Bajon était rentré en France, sans doute dès 1801, et le commissaire du gouvernement Victor Hugues avait installé comme fermier à Beauregard son propre beau-frère, Gauthier. Ce mode de gestion paraissant peu profitable, les copropriétaires se mirent d'accord pour nommer gérant un habitant de Cayenne, Martin, qui adressait en France des traites: $48.000 \mathrm{Fr}$. en mai 1806, 36.000 Fr. en mai 1807, 27.750 Fr. en novembre 1808. Et puis ce fut l'invasion portugaise, dont les destructions a Beauregard furent chiff rées à plus de 168.000 Fr., argent de France. Pendant 6 ans, l'habitation fut de nouveau affermée, mais sans profit; l'indemnité octroyée à la suite du traité avec le Portugal du 7 décembre 1839 ne couvrira que $35,64 \%$ des pertes de 1808 , seules reconnues, et il sera perçu en $1842,59.891$ Fr. 96 en rente $5 \%$, plus 3755 Fr. 99 d'intérêt de ce capital depuis 1840 , date d'effet de la liquidation.

En 1816, on se consultait pour savoir lequel des associés irait assurer la gestion directe, seul moyen d'obtenir le revenu maximum de cette belle habitation, comptant encore 250 esclaves, dont 100 seulement, il est vrai, pouvaient être mis aux cultures. Mais en 30 ans, bien des changements s'étaient produits: Bertrand Bajon était mort

40. Ces inscriptions ont été pour les bénéficiaires une manière de spoliation: en 1805 , le cours de la rente était autour de 60 Fr.; en 1820, de 80 Fr.; des mouvements de Bourse étaient même provoqués par le gouvernement avant ces opérations, où la rente fut toujours comptée au pair.

41. L'inscription au Grand Livre résultant de cette indemnité n'est pas mentionnée dans le dossier. 
et, dès 1822, son neveu et seul héritier, Jean Piquepé, envisagera de vendre sa part; Leroy de la Brière avait laissé un fils, né vers 1785, mais Parisien décidé comme son père; Rouhette de Villeclos, exécuté à Paris sous la Terreur (1er floréal An II), avaient recueilli sa succession en 1805: son frère, Nicolas Rouhette de Monforand, propriétaire rural au Sap, près de Séez (Orne), et sa sœur, Julie, épouse de François Dumont, ancien avocat au Parlement de Paris, devenu membre du Conseil des Anciens, puis chef de division des Baux et Forêts à Bourges, sa ville natale, où il s'était retiré. Finalement, ce fut le fils de Monforand, Maximilien, qui, chargé de toutes les procurations, s'embarqua: il devait faire à Beauregard 2 séjours, l'un de 1817 à 1822, l'autre de 1826 à 1829. C'est alors qu'a été établie par François Dumont (jusqu'à sa mort, en 1827), et conservée, la comptabilité la plus précise, grâce à laquelle il est permis d'évaluer rigoureusement pour les dix années 1817-1826 le taux d'intérêt du capital investi en 1787: pour sa part d'1/8 dans la société (correspondant à la somme une fois versée de 8.163 livres 15 sols, ou 7.940 Fr. 84), Dumont a perçu, net, le total de 14.796 Fr. 82 , soit 1.479 Fr. 68 en moyenne par an, ou $18,63 \%$ du capital.

Encore cette moyenne ne traduit-elle pas le caractère très irrégulier du revenu. D'ailleurs, après 1825 , les bonnes années sont passées: Monforand, de 1826 à 1829, s'attache surtout à rembourser les dettes faites en son absence. Le déficit à peine comblé, les régisseurs des années 1829 à 1837 le creusent de nouveau: Dorniac et Lalanne (1829-1831), Fourcade (1831-1834), gens de Cayenne que Monforand croyait de confiance et habiles, mais malhonnêtes ou que trompaient les économes, comme déjà ils avaient trompé David Flotte, le gérant de 1822 à 1826; après Gasc (1834-1837), envoyé de France et contre lequel s'engagea un interminable procès de peu de profit, on se résolut au système du fermage. En fait, la somme de $12.000 \mathrm{Fr}$. par an prévue dans les différents baux de 1837 à 1848 fut rarement obtenue: Pourjardieu, pour 34 mois, ne versa que 19.000 Fr., le reste étant imputé au compte de débit pour amélioration du fonds; Piquepé, propriétaire d'une moitié, fermier de l'autre, passé en 1840 à Cayenne, y mourut 3 ans plus tard, laissant des dettes considérables, et une veuve qui s'obstina, malgré son imcompétence, à garder l'habitation jusqu'à expiration du bail: 3 ans de fermage semblent alors avoir été perdus sans recours, malgré l'action en 
justice; Emler, avoué de Cayenne, qui prendra l'habitation en 1847, n'aura pas encore payé son dû en 1856 .

A partir de 1848, date de l'émancipation, non seulement les revenus s'effrondrent, mais le fonds lui-même tend à disparaître. Emler, sans esclaves, conserve la ferme à $4.000 \mathrm{Fr}$., mais renonce au bout d'un an (1849). Un administrateur séquestre aux appointements de 1.000 Fr. par an, nommé par le Tribunal de Cayenne, paraît le moyen le moins onéreux de conserver Beauregard dans ce moment incertain, et d'éviter la vente par licitation que réclament les créanciers de la succession Piquepé. On s'estime heureux en France que la gestion alors ne soit pas déficitaire. Quelques revenus - les derniers venant de ces biens - seront obtenus par le bail consenti à Lecomte pour 1856-1865, à raison de 1.600 Fr. par an. Depuis 1830 déjà, on cherchait à vendre: la maison de Cayenne sera cédée en 1865 pour $10.000 \mathrm{Fr}$., puis, en 1866 , l'habitation pour même somme; la pièce qui clôt le dossier, en 1876, concerne la distribution d'un reliquat provenant du paiement de Beauregard. Après l'émancipation, la loi du 30 avril 1849 accordait aux propriétaires une indemnité: il fut d'abord versé en 1850, $30 \mathrm{Fr}$. par esclave (de ceux soumis à capitation, soit 168 à Beauregard), représentant dans l'esprit du législateur une partie du salaire de la première année de ces nouveaux libres; en second lieu, furent attribuées en 2 fractions, dans l'année 1851, des rentes $5 \%$ sur la même base de $30 \mathrm{Fr}$. (de rente, ou $600 \mathrm{Fr}$. de capital, la rente étant au pair) par tête; un prélèvement de $12,5 \%$ sur cette attribution devait servir à constituer le capital de la Banque de la Guyane, prélèvement dont les propriétaires recevraient l'équivalent en actions de la Banque. Les opérations de liquidation furent longues et compliquées: pour 21 esclaves ( $1 / 8$ dans la société) Julie Rouhette $\mathrm{V}^{\mathrm{e}}$ Dumont et, après sa mort, en 1852, sa fille Françoise, épouse de Jules d'Haranguier de Quincerot, reçurent des certificats d'un montant de 10.827 Fr. 35, à convertir obligatoirement en titres de rente $5 \%$, une action de $500 \mathrm{Fr}$. de la Banque de la Guyane, et le reste en diverses sommes donnant 2.166 Fr.

$\mathrm{Au}$ terme de cette longue période de 80 ans, que restait-il aux mains des représentants de Rouhette de Villeclos? Outre les revenus, substantiels, mais qui avaient été perçus de façon capricieuse, le capital se conservait, augmenté, dans divers titres de rente portant 
intérêt à $5 \%$, et dans une action - devenue double en $1864^{43}$ donnant des dividendes. Des formes anciennes du capitalisme, où les relations d'homme à homme tenaient une plus grande place, on avait glissé à des formes de plus en plus impersonnelles: au temps des gérants, le propriétaire lointain tenait à connaitre l'homme chargé de faire du revenu, mais aussi le fonds, l'instrument ${ }^{44}$; au temps des fermiers, ces liens n'ont plus la même nécessité, pourtant le fermier est encore un homme; entre 1850 et 1865, on aboutit à la rente, sous sa forme la plus dépersonnalisée, n'ayant que la sécheresse du chiffre. On s'est d'abord attaché à la description de cette évolution du capitalisme, parce que colonial, bien qu'extérieur à la colonie: c'est le fait saillant du dossier de l'habitation Beauregard.

Les facteurs humains gardent leur importance dans ce dossier surtout avant 1848: les difficultés de l'histoire guyanaise peuvent se suivre à travers celles qui sont particulières à cette habitation. On discerne que, durant la Révolution et l'Empire, malgré l'occupation portugaise de 1808 à 1817 , la Guyane fut un secteur préservé dans l'ensemble des possessions coloniales françaises: point de mouvement des esclaves et par là, point de ruines irréparables comme dans les Antilles. Il suffit de 2 ans de travail à Maximilien de Monforand pour redonner à Beauregard une belle prospérité: de mars 1817 à mars 1818 sont recueillis 73.438 livres de sucre (dont 68.420 de brut), 6.642 gallons de mélasse, 3501 pots de sirop et 1836 de tafia; l'année suivante, les chiffres correspondants sont 124.424 pour le sucre (dont 120.387 de brut), 10.548 pour la mélasse, 336 pour le sirop et 3.903 pour le tafia. A son départ, en octobre 1822, Monforand laisse 62 carrés de cannes en plein rapport et une plantation neuve de 32.600 caféiers. Mais ces efforts restent peu clairvoyants,

42. Pour un capital au pair de 24.430 Fr. Mais il est vrai que la confiance n'étant pas entière dans l'Etat, plusieurs de ces inscriptions de rentes avaient 6té très vite négociées et bien au-dessous du pair.

43. A la suite du vœu émis le 18-I-1863 par l'assemblée générale des actionnaires de la Banque de la Guyane, le capital initial de 300.000 Fr. avait été doublé par décret du 5-VII-1863. Les actionnaires anciens, à qui la réserve était distribúée (250 Fr. 83 par action de $500 \mathrm{Fr}$.) avaient priorité pour souscrire les nouvelles actions. Madame Ve de Quincerot fit le versement complémentaire de 249 Fr. 17 le 16-III-1864.

44. Dans le fonds, on englobe à l'époque les esclaves; ils sont considérés comme instrument, et plus encore par le propriétaire absent qui n'est jamais venu à la colonie. 
parce que seulement conservateurs; ils sont animés de l'esprit du XVIIIe siècle, maintenant condamné. Depuis 1815 , la traite négrière est interdite, mais l'application de cette mesure est laissée à l'initiative personnelle des Gouverneurs, tantôt intransigeants comme Laussat en 1821, tantôt pleins d'indulgence: personne cependant, ni les ministres, ni les gouverneurs, ni les propriétaires, ne pense sérieusement avant 1845 au remplacement de l'esclavage par un système cohérent, capable de concilier les devoirs de l'humanité, les exigences de l'Angleterre et la sauvegarde d'une économie fragile. La décision de l'émancipation en 1848 tombera dans un monde qui, volontairement, aura refusé de s'y préparer.

L'attitude aveugle et raidie des planteurs pourrait trouver son explication dans les difficultés d'ordre fiscal et économique auxquelles ils ont été soumis à cette époque. Jusqu'en 1848, la tendance générale des prix est à la stagnation, ou même à la baisse: le sucre brut de $80 \mathrm{Fr}$. (monnaie coloniale) les 100 livres en 1817, se payait $35 \mathrm{Fr}$. en 1821 à Cayenne; sur la place du Havre, le cours du même sucre était passé de 82 Fr. 50 à 67 Fr. 50 d'aout 1818 à avril 1821, et sur celle de Nantes, de 70 Fr. à 58 Fr. 15 de juin 1819 à mars 1822 . Sans considérer aucunement la conjoncture, l'administration maintenait ses taxes au même niveau: à Cayenne, 1 Fr. 45 (monnaie coloniale) au cent de sucre fabriqué et autant, éventuellement, à la sortie de la colonie; en France, un droit dit "de consommation" payé à raison de 22 Fr. 50 à l'entrée, sur la même quantité. Pour l'économie de plantations, le négoce n'était pas moins dévorant que la fiscalité. Sur la récolte de 1818-1819 à Beauregard, 79.713 livres de sucre brut furent expédiées à la consignation des maisons de commerce Oursel du Havre et Haëntjens frères, de Nantes. Vendus à Cayenne, où les cours varièrent de 70 à $60 \mathrm{Fr}$. le cent, soit $46 \mathrm{Fr}$. 66 à $40 \mathrm{Fr}$. argent de France, ces sucres eussent produit 35.730 Fr. 41 (même monnaie); écoulés à des prix situés entre 82 Fr. 50 et 70 Fr. au Havre et à Paris, ils ne donnèrent au total que 18.199 Fr. 94. La différence tient dans le coulage, c'est-à-dire une perte en poids de règle dans le transport en barriques et qui oscille autour de $10 \%$, dans les frais d'assurance, le frêt (payé à l'arrivée 20 à 22 deniers - la réduction en francs étant faite ensuite - par livre pesant net, majoré pour "chapeau" de $15 \%$ ), la manutention au port (débarquement, transports divers, travail du tonnelier), le magasinage ( $0 \mathrm{Fr} .50$ par barrique et par 
mois), l'expédition, le cas échéant, à Paris, la commission de l'armateur (pour réception, $1 \%$ ad valorem), auxquels s'ajoutent les taxes déjà énumérées (les 22 Fr. 50 aux 100 livres net mentionnés subissent d'ailleurs une majoration de $10 \%$, et le receveur des douanes perçoit, à titre personnel, $1 / 2 \%$, sur ce "droit" de 24 Fr. 75); l'avance de toutes ces sommes étant faite par le négociant, celui-ci retire encore un intérêt de $3 \%$ jusqu'à leur remboursement.

Dans ces conditions, on ne s'étonnera pas que la période du Blocus continental, où n'étaient envoyées en France que des traites, ait été, pendant 2 ans, une des plus avantageuses; on comprendra que la perte en mer des 12.493 livres de sucre chargées le 15 janvier 1818 sur l'Elisa (de la maison Haëntjens) ait été une excellente affaire, la prime d'assurance n'ayant pas eu à supporter les frais habituels. Et on se demandera pourquoi toute la production de l'habitation n'était pas écoulée à C'ayenne; les gérants de Beauregard, et Monforand mieux que tout autre, firent des efforts dans ce sens: en $1817-1818,71.5 \%$ des sucres vendus (sans compter la totalité des sous-produits) l'étaient sur place tant en gros qu'au détail, ce qui représentait un peu plus de 30.000 livres. Ce chiffre était d'ailleurs le maximum possible, en tenant compte des besoins locaux et du frêt étranger alors disponible; si la production augmentait, il était donc impossible de ne pas expédier le surplus en France. On voit l'acuité du problème dès l'année 1818-1819; c'est là ce qui poussera Monforand au développement de la culture du café, qui devait être plus rémunératrice.

A cette évocation sommaire, on mesurera l'importance de ce dossier, qui ne concerne, il est vrai, qu'une seule habitation de la Guyane, mais parmi les premières, sinon la première à cette époque.

C'est encore à la Guyane, hollandaise cette fois, que se rapportent un plan d'une part, divers papiers et un portrait d'autre part, qui nous donnent quelques lueurs sur l'action des Français dans ce domaine étranger. Mis récemment en vente à Paris ${ }^{45}$, ce

45. Par le libraire Roux-Devillas, 12, rue Bonaparte, Paris, 6e. Cf. Catalogue, Nouvelle Série, no 29 , p. 20 , article 327 . 
plan, dressé vers 1770 par l'ingénieur Jan, Daniel Knapp, édité chez de Visscher à Amsterdam, est celui de la colonie du Rio Berbice et de son affluent, le Rio de Canje. Une société de 9 directeurs, parmi lesquels des membres de la famille Dedel, armateurs hollandais connus dès 1750 , avait reçu le privilège de concéder les terres, et encadrait les colons; on compte 93 habitations sur le Rio Berbice et 20 sur le Rio de Canje. L'intérêt de ce document réside dans le nom de plusieurs des colons, incontestablement de souche protestante française: Marret, Balguerie, Fiseaux, Renouard, Giraud, Cazenove, Gaultier.

Ces régions, dont les Anglais s'étaient emparés au cours de la guerre d'Indépendance américaine, furent ensuite occupées par l'expédition que commandait Kersaint. La présence d'un noyau de colons de vieille origine française a pu conduire à l'idée d'un établissement durable: en mai 1782, un gouverneur y était nommé. C'était Louis, Antoine de Lusignan, né à Québec en 1726, devenu après 41 ans de carrière sur mer et dans les divers établissements français d'Amérique, brigadier des armées navales. Son gouvernement s'étendait sur les colonies de Berbice, d'Essequebo et Démérary. Il devait y mourir le 22 juillet 1783 . Son portrait et quelques papiers conservés au château de la Merveillère ${ }^{46}$ font connaître, sans plus, le personnage.

(d suivre)
G. A. Chevalier
G. Debien
L. Dermigny
M. GAUChER
C. Hugues
J. Marion
R. Massio
R. RICHARD

46. Par Thure, près Châtellerault (Vienne). 